\title{
A Comparative ANALYSis ON ROUTING Protocols of Mobile Ad Hoc NeTWORK
}

\author{
Tarikuzzaman Emon, Tarin Kazi and Nazia Hossain \\ Department of Computer Engineering, Stamford University, Dhaka, Bangladesh
}

\begin{abstract}
In this modern era, Mobile Ad hoc Network (MANET) is widely used for its mobility and self-configuring features. Hence, a better security approach is needed for maintaining high level network performance in mobile ad hoc network. This paper presents the comparative analysis of quality of service (QoS) of MANET protocols. In this regard, three types of most widely used protocols, named, Ad hoc On-demand Distance Vector (AODV), Dynamic Source Routing (DSR) and Optimized Link State Routing Protocol (OLSR) have been used to analyze. Therefore, AODV is selected in distributed ad hoc setting for the path discovery. Also, AODV is a reactive improvement of Dynamic Destination Sequence Vector (DSDV) protocol. On the other hand, the number of route broadcast is decreased by source routes on demand in AODV, which is opposite of DSDV algorithm. In this research, the quality of service parameters of network paths are also included to develop trust in the links to defend from various attacks. Moreover, a comparative analysis of delay, congestion, packet losses, transit time between source and destination is illustrated on AODV and DSR. In simulation time, packet drop-outs and link failures for probe packets are examined and analyzed under network attack.
\end{abstract}

\section{KEYWORDS}

MANET, ADOV, DSDV, OLSR, Routing Protocol.

\section{INTRODUCTION}

Ad-hoc mobile nodes are free to move that's why there is no specific network topology. A numbers of routing protocols designed for mobile ad-hoc network to maintain communication among mobile nodes [2]. There are different types of routing protocol- proactive, reactive and hybrid. The objective of this dissertation is to analysis performance of different routing protocols for Mobile Ad-Hoc Networks. Most popular routing protocols are AODV, DSR and OLSR.

Nowadays mobile ad-hoc networks have been growing rapidly according to availability of new generation mobile devices. Since the characteristics of MANET- nodes are mobile. So protocol performance is key issue for any mobile ad-hoc network. In a mobile ad-hoc network implementation of high performance mobile routing protocol is very essential to get better network performance. Ad-hoc network are decentralized and each mobile node can acts as a host and router to communicate with other node.

The aim of this research is to investigate the characteristics of mobile ad-hoc network protocols specially Ad-hoc On-demand Distance Vector (AODV) and Dynamic Source Routing (DSR) and improve their quality of service in several situations. Also Compare to Optimize Link state routing protocols (OLSR) in different mobile ad-hoc network scenarios.

DOI : 10.5121/ijcseit.2014.4404 
In this regard, OPNET is applied to design different mobile ad-hoc network scenarios like node mobility, network load and node density by using popular mobile routing protocols (AODV, DSR, OLSR).

Then it is required to collect OPNET simulated data from different designed network scenarios and analyze simulated data considering MANET routing protocol metrics.

\section{MethodologY}

To evaluate performance of different routing protocol following metrics are considered [3]

\subsection{Average End-to-end Delay}

Average end-to-end delay defines how long it takes for an entire message to completely arrive at the destination from the time the first bit is sent out from the source. It is average of latency for route discovery, interface queues, propagation delay and retransmission delay. [1]

\subsection{Routing Overhead}

Routing overhead is ratio between the numbers of routing packet transmitted for simulation and number of data packet transmitted. [3] This ratio indicates the number of routing control packets required for each data packet transmission.

\subsection{Throughput}

Throughput is the average rate of successful packet delivery from source to destination during simulation [3].

\subsection{Network Load :}

Network load indicates the utilization of link capacity between mobile nodes (amount of traffic or packets/sec passing through it) [3]. If metric value of network load changes very frequently, then it can affect overall mobile network performance.

The goal of this paper is performance analysis of different routing protocols for Mobile Ad-Hoc Networks in different network scenarios. Three types of network metric are used, that is, Network mobility, Network load and Node density, to evaluate routing performance. AODV, DSR and OLSR protocols are considering here for each network scenario. In this project, total 36 scenarios are designed for AODV, DSR and OLSR protocol where the simulation time for each scenario is about 3600 second.

For each protocol (AODV, DSR, OLSR) 12 scenarios- Mobility $(10 \mathrm{~m} / \mathrm{sec}, 20 \mathrm{~m} / \mathrm{sec}, 30 \mathrm{~m} / \mathrm{sec}$ and 40m/sec), Network load (512 bit/sec, $1024 \mathrm{bit} / \mathrm{sec}, 1536 \mathrm{bit} / \mathrm{sec}$ and $2048 \mathrm{bit} / \mathrm{sec}$ ) and Node density (N15, N20, N25 and N30).

Each scenario for node mobility and network load containing 20 nodes. Since mobility is the fundamental basis of mobile ad-hoc network. Random waypoint used here for define mobility speed $(10 \mathrm{~m} / \mathrm{sec}, 20 \mathrm{~m} / \mathrm{sec}, 30 \mathrm{~m} / \mathrm{sec}$ and $40 \mathrm{~m} / \mathrm{sec})$ and node mobility rectangular dimensional area is $1400 \mathrm{~m} \times 1200 \mathrm{~m}$. Packet size for all node mobility scenarios are $1024 \mathrm{bits} / \mathrm{sec}$ and packet rate 5 packet/sec. 
To evaluate performance of network load, MANET traffic generation parameter used here for define different packet size (512bits/sec, 1024bits/sec, 1536bits/sec and 2048bits/sec). Packet inter arrival time $(\mathrm{sec})$ is exp (0.2) and packet rate 5 packet/sec.

To evaluate performance of node density one node density scenario duplicated into 3 scenarios (N15, N20, N25 and N30). Packet size for all node density scenarios are 1024 bits/sec and packet rate 5 packet/sec.

\section{EXPERIMENTAL RESULTS}

There are three types of network scenarios with different parameter designed for simulation. All together 36 scenarios are designed here with different metric values. Node mobility scenarios consider different movement speeds such as $10 \mathrm{~m} / \mathrm{sec}, 20 \mathrm{~m} / \mathrm{sec}, 30 \mathrm{~m} / \mathrm{sec}$ and $40 \mathrm{~m} / \mathrm{sec}$. Network load scenarios consider different packet size such as $512 \mathrm{bits} / \mathrm{sec}, 1024 \mathrm{bits} / \mathrm{sec}, 1536 \mathrm{bits} / \mathrm{sec}$ and $2048 \mathrm{bits} / \mathrm{sec}$. Node density scenarios consider different network size such as 15 nodes, 20 nodes, 25 nodes and 30 nodes. All scenarios simulation time is 3600 seconds.

Illustrative discussion between three types of routing protocol according to node mobility, load and density has been given below:

\subsection{Node Mobility Throughput}

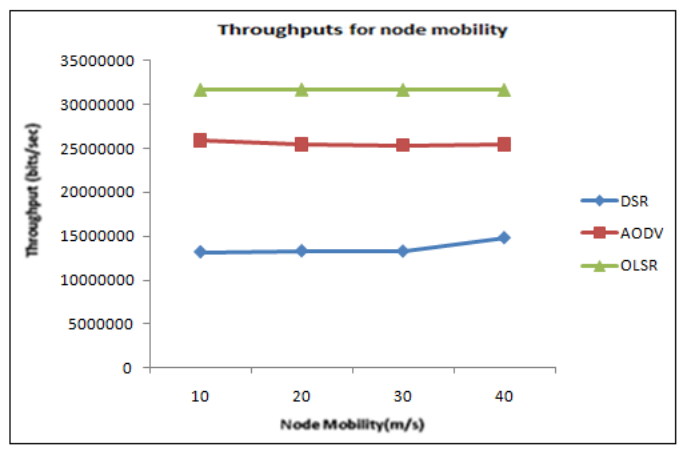

Figure 1. Spam traffic sample

Table 1. Throughputs of Node Mobility

\begin{tabular}{|c|c|c|c|}
\hline Meter/second & DSR & AODV & OLSR \\
\hline 10 & 13194613 & 25918050 & 31698811 \\
\hline 20 & 13313627 & 25438436 & 31725779 \\
\hline 30 & 13271109 & 25337785 & 31684856 \\
\hline 40 & 14791561 & 25429430 & 31675822 \\
\hline
\end{tabular}

Node mobility is basic characteristics of mobile ad-hoc network. The above graph in Figure 1 presenting the node mobility scenarios throughputs of selected routing protocols (AODV, OLSR and DSR). Proactive protocol OLSR shows highest throughput performance among other protocols and DSR showing lowest throughput performance. Since OLSR protocol is table 1 driven and uses multi-point relay broadcast, so it can provides better throughput. AODV protocol capable to form tree to multicast routing so, its performance is good compare with DSR. Since 
DSR is a proactive protocol and uses route discovery process to reach destination, its throughput level is not good for communication latency. OLSR and AODV exchange 'Hello' messages to keep stable connectivity with next hop neighbour nodes.

\subsection{Node Mobility End-to-end Delay}

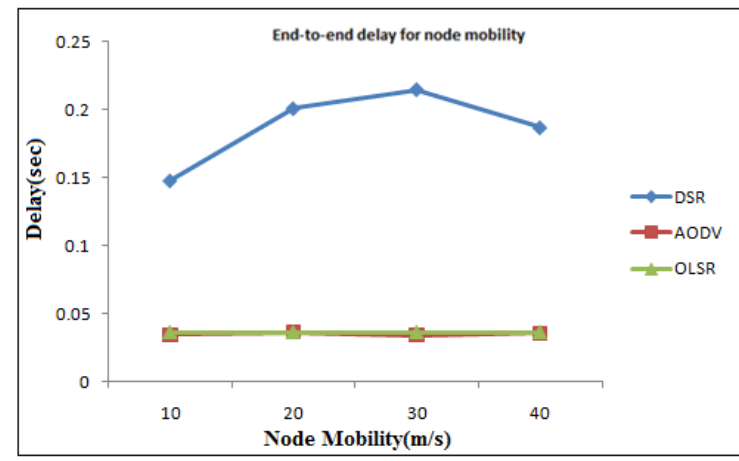

Figure 2. End-to-end delays for node mobility

Table 2. End-to-end delays for different node mobility speed

\begin{tabular}{|c|c|c|c|}
\hline Meter/second & DSR & AODV & OLSR \\
\hline 10 & 0.14764 & 0.034692 & 0.036385 \\
\hline 20 & 0.200927 & 0.036432 & 0.036459 \\
\hline 30 & 0.214962 & 0.034284 & 0.036464 \\
\hline 40 & 0.186824 & 0.03591 & 0.036528 \\
\hline
\end{tabular}

The above graph in Figure 2 presenting node mobility's end-to-end delays for selected routing protocols. OLSR and AODV introducing end-to-end delay between 45-50 milliseconds for different node mobility speed. DSR introducing higher end-to-end delay (150 milliseconds) compare than other routing protocols and end-to-end delay increasing according to change of mobility speed. Since AODV protocol is proactive and capable to form tree for multicast routing, it can exchange routing information faster than DSR. OLSR also capable for multi-point relay and introduce less delay for exchange routing information.

\subsection{Node Mobility Routing Overhead}

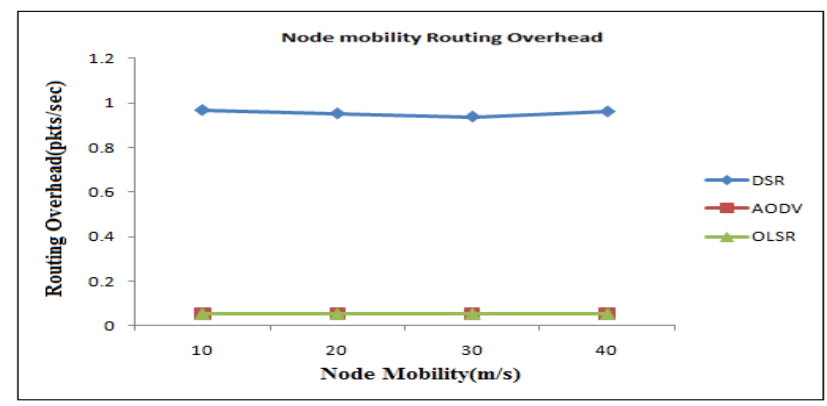

Figure 3. Node mobility routing Overhead 
International Journal of Computer Science, Engineering and Information Technology (IJCSEIT), Vol. 4, No.4, August 2014

Table 3. Routing overhead for different node mobility speed

\begin{tabular}{|c|c|c|c|}
\hline Meter/second & DSR & AODV & OLSR \\
\hline 10 & 0.966 & 0.05302 & 0.053 \\
\hline 20 & 0.951 & 0.0533 & 0.053 \\
\hline 30 & 0.9372 & 0.0532 & 0.053 \\
\hline 40 & 0.9599 & 0.0533 & 0.053 \\
\hline
\end{tabular}

The above graph in Figure 3 presenting node mobility's routing overhead for selected routing protocols. DSR introducing highest routing overhead compare with other two protocols OSLR and AODV. DSR uses route discovery process to select route towards destination that introduces routing overhead. But AODV and OLSR introducing low routing overhead because both protocol uses 'Hello' message to exchange neighbours' routing information. DSR can work perfectly in moderate speed mobility scenario. If mobility speed change frequently, DSR introduce more routing overhead.

\subsection{Node Mobility Network Load}

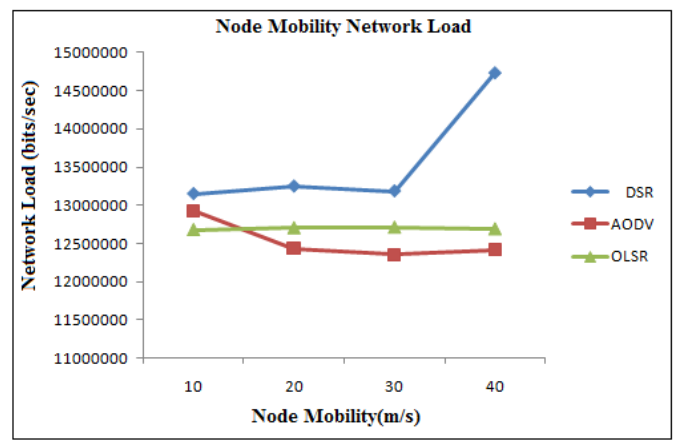

Figure 4. Node Mobility Network Load

Table 4. Node Mobility Network Load

\begin{tabular}{|c|c|c|c|}
\hline Meter/second & DSR & AODV & OLSR \\
\hline 10 & 13150055 & 12930895 & 12676968 \\
\hline 20 & 13248376 & 12433042 & 12701646 \\
\hline 30 & 13185216 & 12353051 & 12708582 \\
\hline 40 & 14732280 & 12420371 & 12686963 \\
\hline
\end{tabular}

The above graph in Figure 4presenting network loads for selected protocols (OLSR, AODV and DSR). DSR introducing high network load compare than other protocols. OLSR shows stable performance for different mobility speed. DSR introduces high network load when mobility speed increase from $30 \mathrm{~m} / \mathrm{s}$ to $40 \mathrm{~m} / \mathrm{sec}$. AODV introducing low network load when node mobility speed increase. Since DSR can work perfectly in moderate speed mobility, so network load increasing rapidly according to speed change. 


\subsection{Network Load (varied packet size) Scenario:}

There are 4 network load scenarios designed to evaluate routing performance. Random away point for each duplicate is $1400 \mathrm{~m} \times 1200 \mathrm{~m}$. MANET traffic generation parameter used to define different packet size (512bits/sec, 1024bits/sec, 1536bits/sec and 2048bits/sec). Inter arrival time $(\mathrm{sec})$ for packet is exp $(0.2)$ and packet rate 5 packet/sec.

\subsection{Varied Network Packet Size Throughputs:}

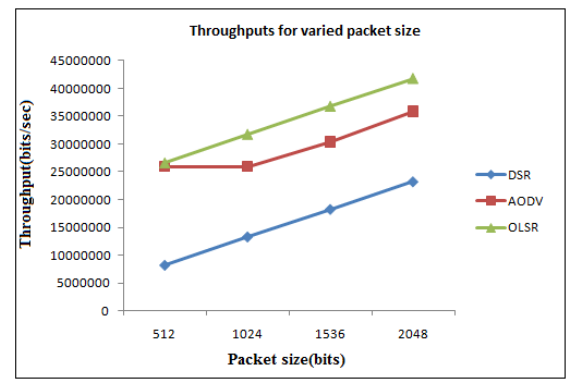

Figure 5. Varied Packet size

Table 5. Varied packet size Throughputs

\begin{tabular}{|c|c|c|c|}
\hline packet size & DSR & AODV & OLSR \\
\hline 512 & 8265657 & 25918050 & 26653261 \\
\hline 1024 & 13363220 & 25918050 & 31771411 \\
\hline 1536 & 18304053 & 30386404 & 36793256 \\
\hline 2048 & 23263544 & 35831837 & 41787249 \\
\hline
\end{tabular}

The above graph in Figure 5 presenting network load (varied packet size) scenarios throughputs of selected routing protocols (AODV, OLSR and DSR). OLSR and AODV providing good throughput compare than DSR. Since mobility speed is low, each protocol can provide better throughput performance if packet size increase. Since OLSR protocol is proactive and uses multipoint relay broadcast, so it can provides batter throughput. Though DSR uses route discovery process to reach destination, its throughput level is good compare than mobility scenario, because DSR spent less time to establish connection with neighbour or next hop nodes.

\subsection{Varied Packet Size End-to-end Delay:}

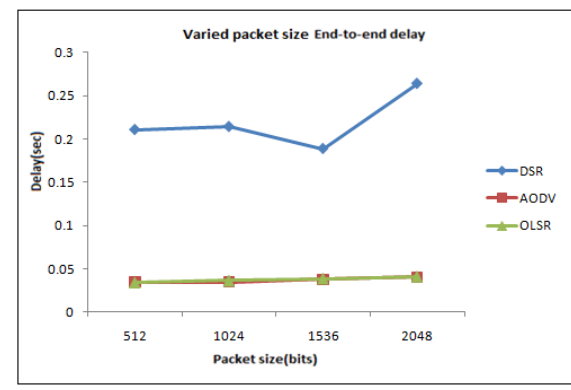

Figure 6. Varied Packet size end-to-end delay 
International Journal of Computer Science, Engineering and Information Technology (IJCSEIT), Vol. 4, No.4, August 2014

Table 6. Varied Packet size end-to-end delay

\begin{tabular}{|c|c|c|c|}
\hline Packet size & DSR & AODV & OLSR \\
\hline 512 & 0.21044 & 0.03469 & 0.03406 \\
\hline 1024 & 0.2139 & 0.03469 & 0.03618 \\
\hline 1536 & 0.1884 & 0.0375 & 0.03854 \\
\hline 2048 & 0.26382 & 0.04012 & 0.04083 \\
\hline
\end{tabular}

The above graph in Figure 6 is presenting end-to-end delays for varied packet size network load. OLSR and AODV introducing low end-to-end delay for different packet size compare than DSR. OLSR uses multi-point relay facilities to broadcast routing information which reduce end-to-end delay.

AODV protocol is capable to form tree during multicast routing. DSR protocol introduces high end-to-end delay during route discovery process. Though mobility speed of network load scenario is moderate, DSR unable to perform well due to varied packet size queuing process.

\subsection{Varied Packet Size Routing Overhead:}

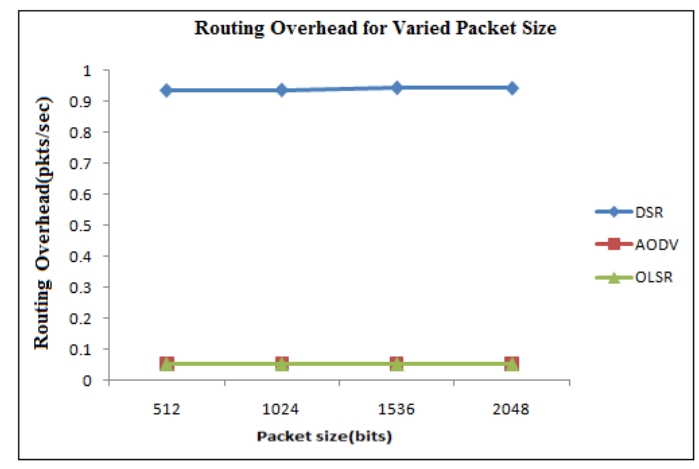

Figure 7. Varied packet size routing Overhead

Table 7. Varied packet size routing Overhead

\begin{tabular}{|c|c|c|c|}
\hline Packet size & DSR & AODV & OLSR \\
\hline 512 & 0.9365 & 0.053 & 0.0526 \\
\hline 1024 & 0.9377 & 0.053 & 0.0526 \\
\hline 1536 & 0.9442 & 0.0532 & 0.0526 \\
\hline 2048 & 0.9429 & 0.0533 & 0.0526 \\
\hline
\end{tabular}

The above graph Figure 7 presenting the routing overheads of network load with varied packet size. Generally DSR introduce low overhead for low mobility scenario, but still introducing highest routing overhead compare with other two protocols OSLR and AODV. AODV and OLSR protocol uses 'Hello' message to exchange neighbour's routing information, that reduce routing overhead. 


\subsection{Network Load for Varied Packet Size:}

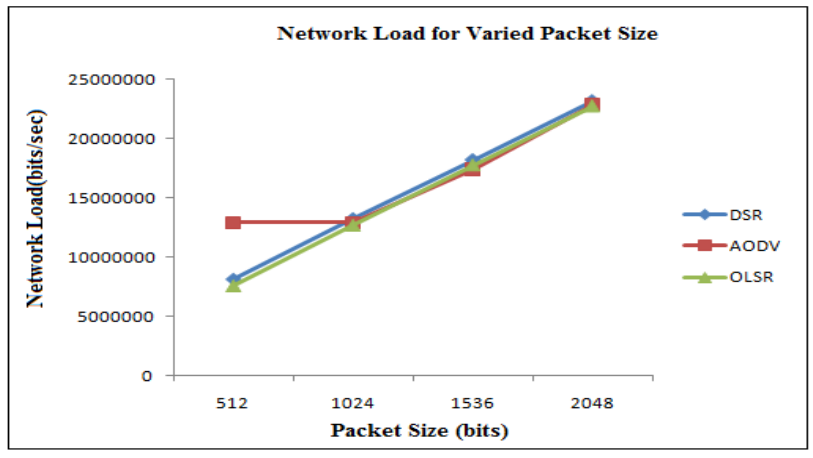

Figure 8. Varied packet size Network Load

Table 8. Varied packet size Network Load

\begin{tabular}{|c|c|c|c|}
\hline Packet size & DSR & AODV & OLSR \\
\hline 512 & 8177526 & 12930895 & 7631579 \\
\hline 1024 & 13277855 & 12930895 & 12728644 \\
\hline 1536 & 18232244 & 17408549 & 17798027 \\
\hline 2048 & 23186119 & 22844991 & 22772754 \\
\hline
\end{tabular}

The above graph in Figure 8 presenting network loads with varied packet size. In varied packet network scenario all protocol performing similar performance. Network load increased regularly due to change of packet size.

\subsection{Node Density Scenario:}

There are 4 node density scenarios designed to evaluate routing performance. Packet size for all node density scenarios are $1024 \mathrm{bits} / \mathrm{sec}$ and packet rate 5 packet/sec.

\subsection{Node Density Throughput:}

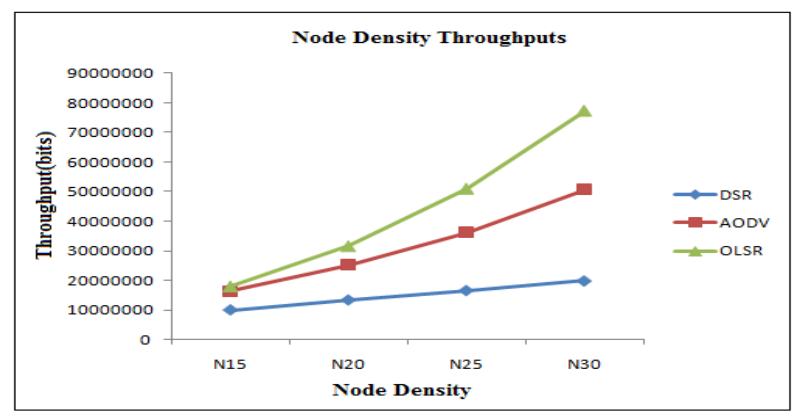

Figure 9. Node density throughputs 
Table 9. Node density throughputs

\begin{tabular}{|c|c|c|c|}
\hline Network Size & DSR & AODV & OLSR \\
\hline N15 & 9948098 & 16351222 & 18132559 \\
\hline N20 & 13380463 & 25325218 & 31675822 \\
\hline N25 & 16656741 & 36241892 & 50999476 \\
\hline N30 & 19965378 & 50720054 & 77225799 \\
\hline
\end{tabular}

The above graph presenting the node density scenarios throughputs of selected routing protocols (AODV, OLSR and DSR). Table driven routing protocol OLSR shows highest throughput performance among other protocols and DSR showing lowest throughput performance. The graph shows that throughputs of all routing protocol increase through, when node density increase. Since OLSR protocol uses multi-point relay technique to broadcast, so density of mobile nodes can provides batter throughput for OLSR. On-demand routing protocol AODV also showing better performance than DSR, because AODV can provide rapid route selection process using uni-cast and multicast routing technique. On other hand DSR uses route discovery process to reach destination, so increase of node density never affect DSR protocol performance.

\subsection{Node Density Throughput:}

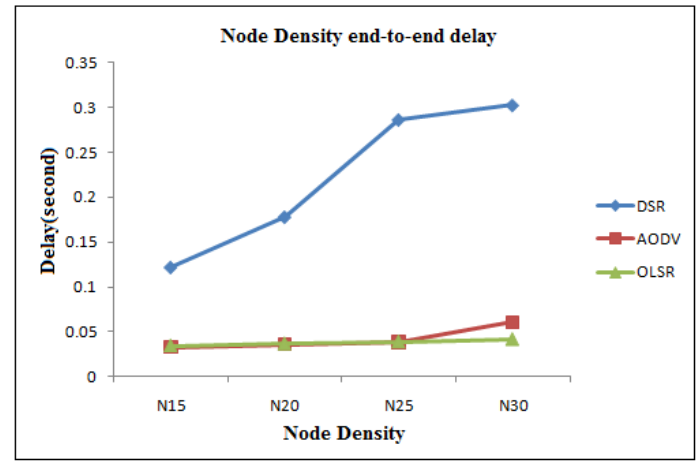

Figure 10. Node Density end-to-end delay

Table 10. Node density end-to-end delay

\begin{tabular}{|c|c|c|c|}
\hline Network Size & DSR & AODV & OLSR \\
\hline N15 & 0.121797 & 0.032539 & 0.034262 \\
\hline N20 & 0.177977 & 0.035767 & 0.036528 \\
\hline N25 & 0.286455 & 0.038447 & 0.038771 \\
\hline N30 & 0.302646 & 0.060549 & 0.041331 \\
\hline
\end{tabular}

The above graph presenting node density scenario's end-to-end delays during routing process. DSR introducing high end-to-end delay compare than other two routing protocol. OLSR introducing lowest end-to-end delay compare than other routing protocol. OLSR uses multi-point relay during routing process, so increase of node density enhance the capability of exchange 'Hello' message and Topology control message perfectly that reduce delay for exchange routing information. Above graph shows that when node density increase from N25 to N30 AODV 
introduces extra delay. Because each node multicast routing information to all surrounding neighbours' node that may introduce additional delay during routing process. On the other hand end-to-end delay of DSR increase when node density increase, because each node maintain a route cache for all possible destination and forward topology change or update information by flooding through whole network that introduce network latency.

\subsection{Node Density Overhead:}

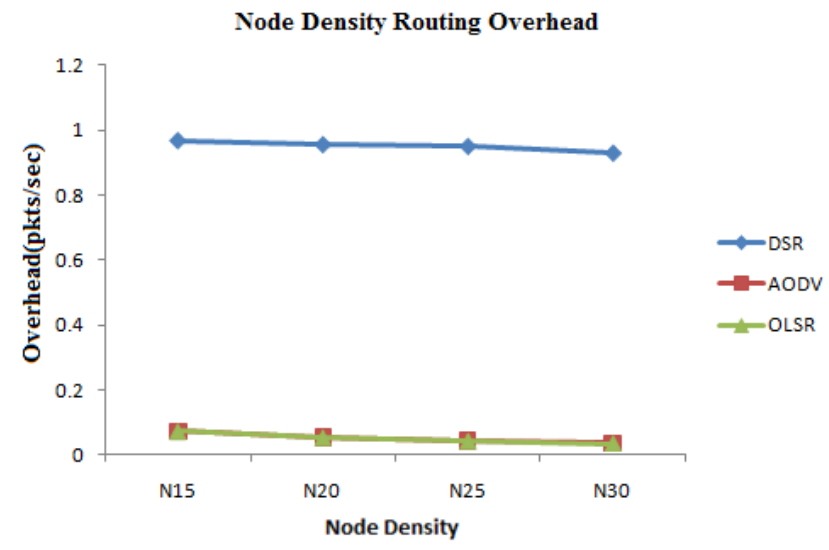

Figure 11. Node Density Routing Overhead

Table 11. Node Density Routing Overhead

\begin{tabular}{|c|c|c|c|}
\hline Network Size & DSR & AODV & OLSR \\
\hline N15 & 0.9661 & 0.07197 & 0.07191 \\
\hline N20 & 0.9542 & 0.0532 & 0.0526 \\
\hline N25 & 0.9487 & 0.0421 & 0.0416 \\
\hline N30 & 0.9285 & 0.03608 & 0.0344 \\
\hline
\end{tabular}

The above graph presenting node density scenario's routing overhead during routing process. DSR introducing highest routing overhead compare than OSLR and AODV. DSR uses route discovery process before select a route toward destination, which is the reason for high routing overhead but overhead level is stable for all topological change. AODV and OLSR introducing low routing overhead during routing process to exchange neighbours' routing information using 'Hello' message. OLSR and AODV can exchange 'Hello' message and Topology control message perfectly with neighbour nodes, when node density increase within network. 


\subsection{Node Density Network Load:}

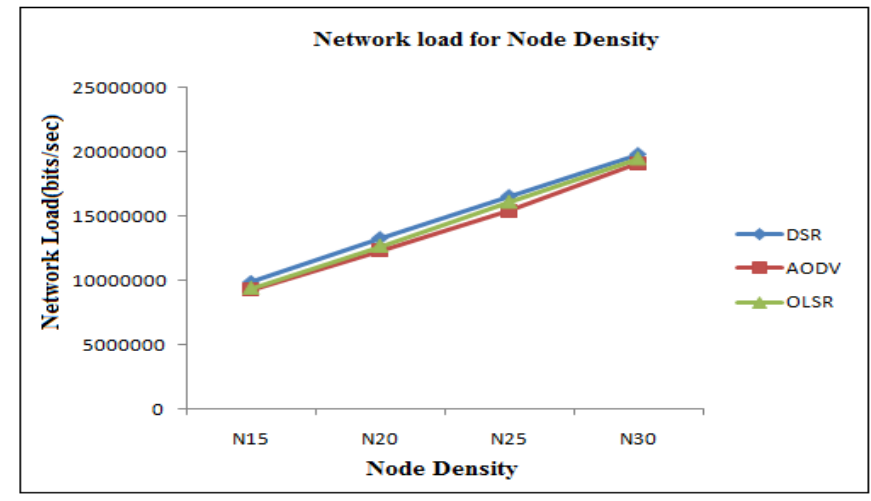

Figure 12. Node Density Network Load

Table 12. Node Density Network Load

\begin{tabular}{|c|c|c|c|}
\hline Node Density & DSR & AODV & OLSR \\
\hline N15 & 9914678 & 9304493 & 9393939 \\
\hline N20 & 13319293 & 12336487 & 12686963 \\
\hline N25 & 16569224 & 15441027 & 16098506 \\
\hline N30 & 19818532 & 19117896 & 19491134 \\
\hline
\end{tabular}

The above graph presenting network loads for node density scenario. All protocols network load increasing according to node density increase. DSR introducing higher network load and AODV introducing low network load. After that process continues repeatedly for Network Load and Node Density.

\section{Comparative Analysis}

In these paper three types of MANET scenario considered for simulation. 4 node mobility scenarios with different movement speed, 4 Network load scenarios with different packet size and 4 Node density scenarios with different network size. Each protocols performance can be analyse according to performance metrics and key findings from whole study are given below:

\subsection{Throughut:}

AODV and OLSR provide better network throughput for three different network scenarios compare than DSR protocol. Since OLSR protocol support multi-point relay, it introduce high throughput for all types of network scenarios. Throughput performance of AODV also good for all scenarios compare than DSR throughput performance. But DSR fail to achieve standard throughput performance for any types of network scenarios compare than other protocols. All protocol performs comparatively well in network load (varied packet size) scenarios because mobility speed was low. On other hand in node density scenarios all routing protocol increase throughput, when node density increase. 


\subsection{End-to-end Delay:}

DSR introduces higher end-to-end delay in all scenarios compare than OLSR and AODV protocol delay. In mobility scenarios end-to end delay increase when mobility speed increase. Though, DSR perform well at moderate node speed but unable to perform well in network load scenarios due to varied packet size. In node density scenarios OLSR perform well compare than other two protocols. When node density increase OLSR reduce end-to-end delay due to enhancement of exchange routing capability. On other hand DSR introduce additional delay when node density increase.

\subsection{Routing Overhead:}

DRS introduce highest routing overhead in all scenarios compare than OLSR and AODV. Since DSR only capable to perform with moderate speed, so in mobility scenario it cost additional overhead due to mobility speed change. AODV and OLSR uses 'Hello' message for exchange routing information that is why both protocol introduces low routing overhead compare than DSR.

\subsection{Network Load:}

In node mobility scenario OLSR performed stable performance, but DSR introduces high network load due to increase of node mobility. AODV reduce network load when mobility of nodes increase. All protocol increase network load when packet size and node density increase in network load scenarios and node density scenarios respectively.

\section{Conclusion}

This paper is representing performance analysis of different routing protocols for Mobile ad-hoc network. According to this paper Researchers tried to discuss all common mobile routing protocol with their features and mode of operation. Here OPNET network simulator has been used to evaluate performance. Since OPNET is a study version of simulation tool, so it has limited choice of routing protocol. From OPNET protocol parameter option, OLSR, DSR and AODV routing protocols has been selected to performance evaluation. So, it was really hard to evaluate routing performance for all discussed (within this paper) routing protocols perfectly in realistic scenario.

\section{ACKNOWLEDGEMENTS}

First of all we acknowledge to Almighty for completing this research successfully. Then we are grateful to all of the authors individually. We would like to thank our parents, friends for their invaluable suggestions and critical review of our thesis.

\section{REFERENCES}

[1] Stefano Basagni, Marco Conti, Silvia Giordano, Ivan Stojmenovic, (2004) "Mobile ad hoc networking”, Wiley-IEEE Press

[2] Mehran Abolhasan, Tadeusz Wysocki, Eryk Dutkiewicz, ( 2003 )“A review of routing protocols for mobile ad hoc networks" ,Telecommunication and Information Research Institute, University of Wollongong, Wollongong, NSW 2522, Australia,Motorola Australia Research Centre, 12 Lord St., Botany, NSW 2525, Australia 
[3] S.R. Biradar, Hiren H D Sarma, Kalpana Sarma, S.K. Sarker and Puttamadappa C, (2009 )“ Performance Comparison of Reactive Routing Protocols of MANETs using Group Mobility Model”, International Conference on Signal Processing Systems, IEEE publisher, pp192-195,

\section{Authors}

Tarikuzzaman Emon received his B.Sc. degree in Computer Science and Engineering from East West University, Dhaka, Bangladesh in 2006, M.Sc. degree in Computer Networking from London Metropolitan University, London, U.K in 2011 and PG.Cert. in Computer System and Networking from University of Greenwich, London, U.K in 2010. He was serving as an IT Field Technician in the IT division at Pret-A-Manager, London, UK from 2007 to 2012. He is serving as a Lecturer in the department of Computer Science \& Engineering at Stamford Un iversity Bangladesh, Dhaka, Bangladesh. His field of research interest includes Computer Networking, wireless mobile communication, Image Processing and Pattern Recognition.

Tarin Kazi has been received her B.Sc Engineering Degree from Ahsanullah University of Science \& Technology, Bangladesh. She is serving as a Lecturer in the department of Computer Science \& Engineering at Stamford University Bangladesh, Dhaka, Bangladesh.

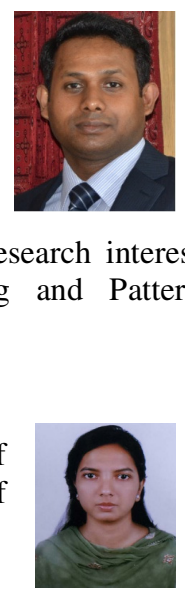

Nazia Hossain has been received her B.Sc Engineering Degree from Khulna University of Engineering \& Technology, Bangladesh. She is now full-time lecturer in Stamford University Bangladesh. And, continuing her graduate study in M.Sc. Engineering in Bangladesh University of Engineering and Technology.

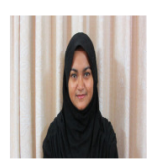

